\title{
High performance computing in astrophysics. The organic formation in protostellar disc
}

\author{
I. Kulikov \\ Institute of Computational Mathematics and Mathematical Geophysics SB RAS \\ *e-mail:kulikov@ssd.sscc.ru
}

Key word: high performance computing, computational astrophysics, protostellar disc, chemodynamics

Abstract: In this talk, a novel computation technique for numerical simulations of astrophysics structures at the Peta- and Exascale supercomputers is described. The co-design of parallel numerical algorithms for astrophysical simulations is described in detail. The hydrodynamical numerical model for the astrophysical simulation, numerical methods for solving the hyperbolic equations and brief description of parallel implementation of the gooPhi code are described. The results of numerical experiments of simulations of organic formation in protostellar disk are presented.

Acknowledgements: The research work was supported by the Grant of the President of Russian Federation for the support of young scientists number MK - 1445.2017.9, RFBR grants 18-01-00166 and 18-07-00757. 